\title{
A LIFE-THREATENING CONDITION AT BIRTH: MATERNAL DISTRESS ONE YEAR AFTER
}

M.F. Coletti, F. Bevilacqua, L. Aite, B. Caravale, C. De Marchis, F. Piersigilli, A. Braguglia, A. Dotta, P. Bagolan

Medical and Surgical Neonatology, Bambino Gesù Children's Hospital, Rome, Italy

Background and aims: In newborns, life-threatening conditions may cause long lasting parental reactions. The stress experienced by parents due to early separation and hospitalization may alter their response to the infant.

Our prospective study aimed to evaluate maternal stress one year after the birth of newborns hospitalized for high risk condition.

Methods: Mothers of newborn babies hospitalized at birth because of severe prematurity $<32$ weeks GA (PT), major congenital malformations (MCM) or severe congenital hearth disease (CHD) were asked to fill the Parenting Stress Index-Short Form (PSI-SF) one year after delivery. In the same assessment child neuropsychological development was evaluated by Bayley Scale (Bayley-III).

We analyzed correlations between the PSI-SF scores and diagnosis, length of hospital stay, neurodevelopmental outcome, genetic syndrome, mother's education and socioeconomic status by t-Test and linear regression analysis $(\mathrm{p}<0.05)$.

Results: 121 mothers and 129 children (41 PT, 39 MCM, 49 CHD) were recruited in the study.

Mothers obtained a mean PSI-SF total score within normal range. $17 \%$ of mothers had at least one subscale in the pathological range (above $85^{\circ}$ centile). Level of parenting stress was significantly correlated with length of hospital stay, child motor development and maternal education.

Conclusions: One year after delivery the vast majority of mothers showed parenting stress within normal range regardless of the medical risk condition of the newborn. According to our data risk factors for increased parenting stress at one year seem to be lower maternal educational level, abnormal motor development and length of hospitalization. 